\title{
\begin{tabular}{l|l} 
Mitraries & DSpace@MIT
\end{tabular}
}

\author{
MIT Open Access Articles
}

\section{Characterizing Dissipation in Fluid-Fluid Displacement Using Constant-Rate Spontaneous Imbibition}

The MIT Faculty has made this article openly available. Please share how this access benefits you. Your story matters.

Citation: Characterizing Dissipation in Fluid-Fluid Displacement Using Constant-Rate Spontaneous Imbibition B.TK. Primkulov, J.Y.Y. Chui, A.RA. Pahlavan, C.WW. MacMinn, and R. Juanes Phys. Rev. Lett. 125, 174503 @ 2020 American Physical Society.

As Published: 10.1103/PHYSREVLETT.125.174503

Publisher: American Physical Society (APS)

Persistent URL: https://hdl.handle.net/1721.1/132991

Version: Final published version: final published article, as it appeared in a journal, conference proceedings, or other formally published context

Terms of Use: Article is made available in accordance with the publisher's policy and may be subject to US copyright law. Please refer to the publisher's site for terms of use. 


\title{
Characterizing Dissipation in Fluid-Fluid Displacement Using Constant-Rate Spontaneous Imbibition
}

\author{
B. K. Primkulov $\odot,{ }^{1}$ J. Y. Y. Chui $\odot,{ }^{1}$ A. A. Pahlavan $\odot,{ }^{2}$ C. W. MacMinn $\odot,{ }^{3}$ and R. Juanes $\oplus^{1, *}$ \\ ${ }^{1}$ Massachusetts Institute of Technology, Cambridge, Massachusetts 02139, USA \\ ${ }^{2}$ Princeton University, Princeton, New Jersey 08544, USA \\ ${ }^{3}$ University of Oxford, Oxford OX1 3PJ, United Kingdom
}

(Received 23 June 2020; accepted 23 September 2020; published 23 October 2020)

\begin{abstract}
When one fluid displaces another in a confined environment, some energy is dissipated in the fluid bulk and the rest is dissipated near the contact line. Here we study the relative strengths of these two sources of dissipation with a novel experimental setup: constant-rate spontaneous imbibition experiments, achieved by introducing a viscous oil slug in front of the invading fluid inside a capillary tube. We show that a large fraction of dissipation can take place near the contact line, and rationalize the observations by means of a theoretical analysis of the dynamic contact angles of the front and back menisci of the oil slug. Our results bear important implications for macroscopic descriptions of multiphase flows in microfluidic systems and porous media.
\end{abstract}

DOI: 10.1103/PhysRevLett.125.174503

Many of our daily experiences involve one fluid displacing another on a solid surface: from cooking oil spreading on a frying pan to paper absorbing ink [1,2] and tea flowing up a biscuit [3]. In all of these examples, capillarity drives the flow as energy dissipates within the fluid bulk and near the contact line (the intersection of the fluid-fluid interface with the solid surface). While dissipation in the fluid bulk is purely viscous, dissipation near the contact line is not yet fully understood [4-14]. Characterizing what fraction of energy is lost in each region is a nontrivial task; the dynamics of contact lines remains in many respects unresolved and continues to challenge our descriptions of multiphase flow $[4,5,10,15]$.

In this work, we unambiguously separate contact-line and bulk dissipation and map out their relative importance in a simple fluid-fluid displacement system. This is challenging since the dynamics of moving contact lines is nonlinear and rate dependent: the macroscopic contact angle $\theta$ at which the fluid-fluid interface meets the solid surface changes with the rate of displacement, and dissipation at the contact line, in turn, changes with $\theta[16]$. The dynamics of moving contact lines has traditionally been studied through two classes of experiments: (i) constantrate displacement under an external force (e.g., dip coating $[17,18]$, forced displacement in capillary tubes $[19,20])$ and (ii) spontaneous, variable-rate displacement (e.g., spreading of a droplet on a solid surface [21,22], imbibition of a liquid into a capillary tube [23-29]).

Here, we present an alternative experimental setup whose novelty is the result of combining, for the first time, three key ingredients: (i) moving contact lines, (ii) a confined geometry, and (iii) spontaneous, constant-rate interfacial motion. Although the dynamics of the moving contact lines was first properly described by Voinov [30] and Cox [31], most studies have focused on unconfined configurations such as spreading of liquid drops on solid surfaces [5,15,32]. Confinement increases the ratio of the interfacial area (solid-fluid and fluid-fluid) to bulk volume, often by orders of magnitude, which raises a fundamental question about the balance among different dissipation sources. While many studies have analyzed the importance of the different contributions to energy dissipation in the context of spontaneous imbibition of a liquid displacing air, as described by the Lucas-Washburn law [1,33], bulk viscous dissipation dominates except at early times [34]. What sets our experimental setup apart from previous studies is that it allows us to achieve constant-rate imbibition, and therefore keep the ratio of the different dissipation contributions fixed throughout each experiment. This allows us to unambiguously extract the sources of dissipation in the different regimes and construct a phase diagram describing the ratio of the energy that is dissipated at the contact line.

Our experimental setup is built upon the classical case of spontaneous imbibition into a capillary tube. By exposing one end of a horizontal capillary tube to a silicone oil reservoir, oil spontaneously wets the capillary ["classical imbibition," Fig. 1(a)]. The position of the oil front $(z)$ mostly follows Washburn's scaling $\left(z \sim t^{1 / 2}\right)$ [33]. The mechanism behind the slowing of the liquid front is well understood: the capillary driving force remains nearly constant, while viscous resistance increases in proportion to $z$. We modify this setup to achieve constant-rate spontaneous imbibition by restricting the viscous resistance to an oil slug of fixed length ["constant-rate imbibition," Fig. 1(b)]. We place a silicone oil (Sigma-Aldrich) slug of viscosity $\mu_{o}$ and length $l$ into a hydrophilic glass tube 

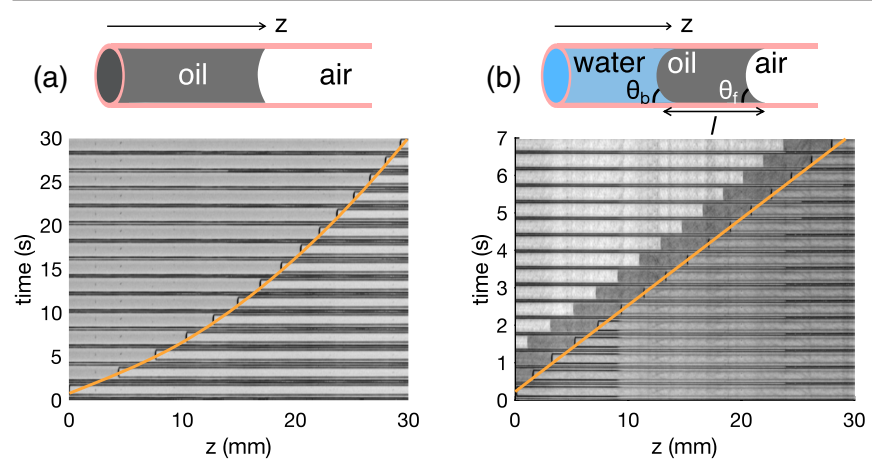

FIG. 1. Experimental snapshots of (a) the classical spontaneous imbibition $\left(z \sim t^{1 / 2}\right)$ of $50 \mathrm{cSt}$ silicone oil in a capillary tube and (b) constant-rate spontaneous imbibition $(z \sim t)$ of water with a $50 \mathrm{cSt}$ silicone oil slug precursor.

(untreated Hilgenberg $\mathrm{GmbH}$ borosilicate glass 3.3), and then expose the end with the slug to a reservoir of water with viscosity $\mu_{w}$. The bulk viscous resistance is then proportional to $\mu_{o} l+\mu_{w} z$; when $\mu_{o} l \gg \mu_{w} z$, the slug moves at a constant rate that can be controlled by tuning $l$ and/or $\mu_{o}$. In our experiments, the length of the oil slug does not change as water penetrates the tube, which implies that the oil slug does not leave a film of oil behind [35-37]. We include further experimental details in the Supplemental Material [38].

In contrast with classical imbibition, the oil slug in our experiments has two menisci: one at the front (oil-air) and one at the back (water-oil) [Fig. 1(b)]. The contact angles of these two menisci are expected to change with the contactline speed, and we use the term "dynamic contact angle" for angles at nonzero speeds. We denote the dynamic contact angles of the back and front menisci as $\theta_{b}$ and $\theta_{f}$, and their respective static-advancing values as $\theta_{b, a}$ and $\theta_{f, a}$. Each individual experiment has a fixed speed and thus fixed dynamic contact angles. To probe the dynamics of the system at different spontaneous contact-line speeds, we span a wide range of slug viscosities and lengths, with $\mu_{o} \in$ $\{48,485,970\} \mathrm{mPa}$ and $l \in[2,14] \mathrm{mm}$. We characterize the nominal ratio of viscous to capillary forces in each experiment through the capillary number $\mathrm{Ca} \equiv\left(\mu_{o} \dot{z} / \gamma_{o}\right)$, where $\dot{z}$ is the slug speed and $\gamma_{o}$ the surface tension of the oil. We plot $\mathrm{Ca}$ against the ratio of tube radius $R$ to slug length $l$ in Fig. 2(a), where 44 constant-rate imbibition experiments collapse onto a single curve. While each individual experiment is constant rate, the nonlinear global trend emerges from the dynamics near the contact lines. We begin to rationalize this trend through force balance.

Constant-rate imbibition is governed by the balance of bulk viscous resisting force $\left(F_{\text {bulk }}\right)$ and capillary driving force $\left(F_{\text {cap }}\right)$. The bulk viscous force can be calculated from the drag on the tube walls by assuming classical Poiseuille flow (see Supplemental Material [38]) as $F_{\text {bulk }}=2 \pi R\left[l\left(4 \mu_{o} / R\right)+z\left(4 \mu_{w} / R\right)\right] \dot{z}$. Since $\left(\mu_{w} z / \mu_{o} l\right) \in$ $[0.001,0.2]$ in our experiments, we neglect the viscous pressure drop within the water phase and the expression for $F_{\text {bulk }}$ reduces to

$$
F_{\text {bulk }}=8 \pi \mu_{o} l \dot{z} .
$$

The capillary driving force can be expressed through the dynamic contact angles of the back and front menisci:

$$
F_{\text {cap }}=2 \pi R\left(\gamma_{o w} \cos \theta_{b}+\gamma_{o} \cos \theta_{f}\right),
$$

where $\gamma_{o w}$ is the oil-water interfacial tension. For quasistatic displacement in the absence of gravity, $F_{\text {cap }}$ and $F_{\text {bulk }}$ must balance to yield the speed of the oil slug, $\dot{z}=\left(R / 4 \mu_{o} l\right)\left(\gamma_{o w} \cos \theta_{b}+\gamma_{o} \cos \theta_{f}\right)$, which in dimensionless form reads

$$
\mathrm{Ca}=\left(\frac{\gamma_{o w}}{\gamma_{o}} \cos \theta_{b}+\cos \theta_{f}\right) \frac{R}{4 l} .
$$

To fully resolve Eq. (3), we need to know how $\theta_{b}$ and $\theta_{f}$ evolve with $\mathrm{Ca}[4,5,15]$. When the solid surface is perfectly
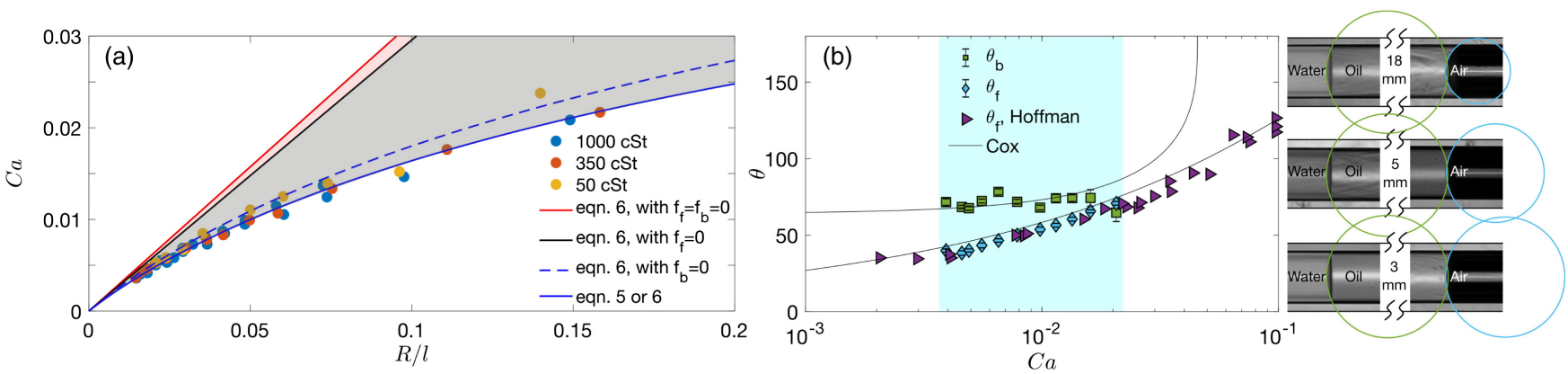

FIG. 2. (a) Constant-rate imbibition experiments for $\mu_{o} \in\{48,485,970\} \mathrm{mPas}$ and $l \in[2,14] \mathrm{mm}$. Solid lines are theoretical predictions of constant-rate imbibition after accounting for dissipation sources within the oil slug. The experimental data is captured accurately by Eq. (5) [or equivalently Eq. (6)]. (b) Measurements of $\theta_{f}$ (blue diamonds) and $\theta_{b}$ (green squares) during constant-rate imbibition were done using a microscope, with typical snapshots for slugs of different lengths (and thus Ca) displayed beside the figure. The solid lines show the generalized Cox relation [31] with $\Gamma=6.9$, and purple triangles indicate the data from Hoffman [19]. The blue shaded region indicates the range of $\mathrm{Ca}$ in our constant-rate imbibition experiments. 
smooth and homogeneous, both angles are expected to follow the generalized Cox equation [31], which can be written as

$$
g(\theta, M)-g\left(\theta_{a}, M\right)=\mathrm{Ca},
$$

where $\Gamma=\ln \left(R / h_{\text {micro }}\right), h_{\text {micro }}$ is the microscopic cutoff length near the contact line, $M$ is the ratio of the defending to invading fluid viscosities, and the function $g(\theta, M)$ is defined in the Supplemental Material [38]. Indeed, when using $M=$ 0 for the oil-air interface, $M=1000$ for the water-oil interface, and $h_{\text {micro }} / R=10^{-3}(\Gamma=6.9)$ for both [31], the generalized Cox equation produces good agreement with our experimental measurements of $\theta_{f}$ and $\theta_{b}$ [Fig. 2(b)]. Although Eqs. (3) and (4) can be used to reproduce the constant-rate imbibition trend in Fig. 2(a), we seek further simplifications of Eq. (4) for the two menisci. First, we take $\theta_{b}=72^{\circ}$. This is justified since both $\theta_{b}$ measurements and the generalized Cox trend in Fig. 2(b) appear to be approximately constant within the Ca range of our constant-rate imbibition experiments. Second, we note that Eq. (4) simplifies greatly for the oil-air meniscus: when $M \ll 1$, it reduces to the commonly used Cox-Voinov relation $\theta_{f}^{3}=\theta_{f q}^{3}+9 \Gamma \mathrm{Ca}[30,31]$. This further reduces to $\theta_{f}=$ $(9 \Gamma \mathrm{Ca})^{1 / 3}$ since silicone oil wets the glass surface completely $\left(\theta_{f, a}=0^{\circ}\right)$. Therefore, after using the expansion $\cos \theta_{f}=$ $1-\theta_{f}^{2} / 2+O\left(\theta_{f}^{4}\right)$ and the Cox-Voinov expression, Eq. (3) yields

$$
\mathrm{Ca}=\left[\frac{\gamma_{o w}}{\gamma_{o}} \cos \theta_{b}+1-\frac{1}{2}(9 \Gamma \mathrm{Ca})^{2 / 3}\right] \frac{R}{4 l},
$$

which accurately reproduces the experimental trend [Fig. 2(a)]. Note that the generalized Cox relation predicts approximately constant $\theta_{b}$ within the $\mathrm{Ca}$ range of our experiments for any liquid pair as long as $M \gg 1$ and $\theta_{b, a} \lesssim 64^{\circ}$.

We can now use this theoretical description of constantrate imbibition [Eq. (5)] to evaluate the contributions of the two moving contact lines to the macroscopic trend in Fig. 2(a). It is important to distinguish between the two menisci in Fig. 1(b), because wettability plays a key role in how they interact with surface defects. The water-oil interface is in partial wetting, and can experience pinning at surface defects [7]; whenever $\theta_{b}<\theta_{b, a}$, interfacial forces at the contact line are in static balance. This balance no longer holds when $\theta_{b}>\theta_{b, a}$, and the contact line sets in motion. We define the dynamic contact-line force at the back meniscus as $f_{b}=\gamma_{o w}\left(\cos \theta_{b, a}-\cos \theta_{b}\right)$. We measure $\theta_{b, a} \approx 64^{\circ}$, and thus $f_{b} \approx 0.13 \gamma_{o w}$. In contrast, the oil-air interface is in complete wetting, and is not sensitive to most surface defects [7]. We define $f_{f}=\gamma_{o}\left(\cos \theta_{f, a}-\cos \theta_{f}\right)$ in analogy to the water-oil meniscus. Recall that $\theta_{f, a}=0^{\circ}$. Then, the force at the front meniscus reduces to $f_{f}=\left(\gamma_{o} / 2\right)(9 \Gamma \mathrm{Ca})^{2 / 3}$. We can then rewrite Eq. (5) through the dynamic contact-line forces,

$$
\frac{4 l}{R} \mathrm{Ca}+\frac{f_{b}}{\gamma_{o}}+\frac{f_{f}(\mathrm{Ca})}{\gamma_{o}}=1+\frac{\gamma_{o w}}{\gamma_{o}} \cos \theta_{b, a},
$$

where "driving" terms are grouped on the right-hand side, and "resisting" terms are grouped on the left-hand side. Equation (6) is equivalent to Eq. (5), but its form is convenient for inferring the relative importance of $f_{b}$ and $f_{f}$ to the overall trend in Fig. 2(a). If there were no dynamic contact-line forces at the two menisci $\left(f_{f}=f_{b}=0\right)$, the equation of motion would reduce to Eq. (3) with $\theta_{b}=\theta_{b, a}$ and $\theta_{f}=\theta_{f, a}$. This scenario corresponds to the red line in Fig. 2(a). If we now remove the dynamic contact-line force at the front meniscus only, Eq. (6) would reduce to Eq. (5) without term $\left(\gamma_{o} / 2\right)(9 \Gamma \mathrm{Ca})^{2 / 3}$, corresponding to the black line in Fig. 2(a). These comparisons suggest that: (i) neglecting the dynamic contact-line forces produces a trend with a significant qualitative and quantitative disagreement with the experiments in Fig. 2(a), (ii) nonlinearity in constantrate imbibition comes from the dynamic contact-line force at the front meniscus, (iii) the contribution of $f_{b}$ to the overall trend in Fig. 2(a) is relatively small [see Eq. (6) with $f_{b}=0$ in Fig. 2(a)], with $2<f_{f} / f_{b}<8$ within the $\mathrm{Ca}$ range of our experiments.

Although our experiments are in spontaneous imbibition, our results are also relevant to forced imbibition. Addition of an external force would not change the sources of dissipation within the moving slug. There are only three dissipative forces in our system: bulk viscous force and contact-line forces at the two menisci. The energy dissipation in the bulk is $\Phi_{\text {bulk }}=8 \pi \mu_{o} l \dot{z}^{2}$, again assuming Poiseuille flow and $\mu_{o} l \gg \mu_{w} z$. The dissipation due to dynamic contact-line forces is $\Phi_{\mathrm{cl}}=2 \pi R\left(f_{f}+f_{b}\right) \dot{z}$. We can map the relative magnitudes of $\Phi_{\text {bulk }}$ and $\Phi_{\mathrm{cl}}$ during arbitrary motion of the oil slug. Figure 3 shows a phase diagram where spontaneous imbibition [Eq. (5)] separates regions where an external force either "pushes" the slug to move faster or "pulls" it to move slower than the spontaneous rate. The ratio of contact line to total dissipation within the moving slug is $\Xi=\Phi_{\mathrm{cl}} /\left(\Phi_{\mathrm{cl}}+\Phi_{\text {bulk }}\right)$, which is equivalent to

$$
\Xi=\frac{f_{b}+f_{f}}{f_{b}+f_{f}+\frac{4 l}{R} \mathrm{Ca} \gamma_{o}},
$$

and can be alternatively derived by considering dissipative forces within the system (contact line vs total). The colormap in Fig. 3 represents different values of $\Xi$ in Eq. (7). A surprisingly large fraction of the dissipation (between $20 \%$ for $14 \mathrm{~mm}$ slugs and $50 \%$ for $2 \mathrm{~mm}$ slugs) occurs in the vicinity of the contact line in our experiments. The values of $\Xi$ in Fig. 3 are within the Ca range of our experiments. However, it is important to note what would happen in the upper and lower bounds of $\mathrm{Ca}$ in Fig. 3. In the upper bound $(\mathrm{Ca}>0.02)$, our approximation of constant $\theta_{b}$ would no 


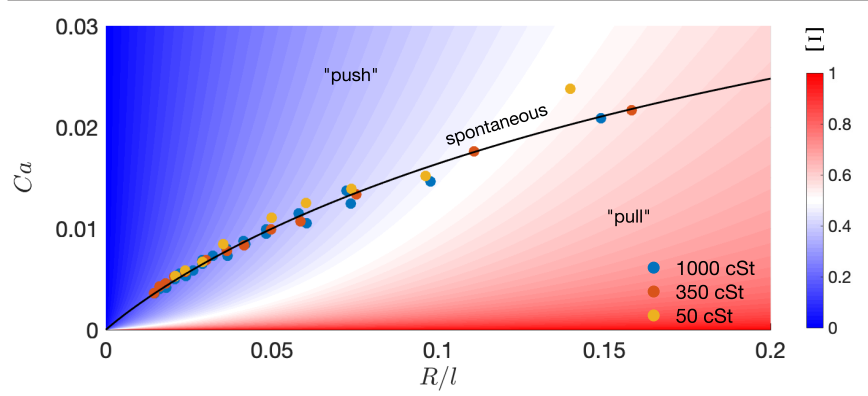

FIG. 3. Phase diagram of forced, rate-controlled imbibition of viscous oil slugs. An external force is needed to move the slug at higher $\mathrm{Ca}$ (push) or lower $\mathrm{Ca}$ (pull) than the spontaneous rate predicted by Eq. (6) (black solid line). The color of the $R / l-\mathrm{Ca}$ space represents the ratios of contact line to total dissipation in such moving slugs, $\Xi=\Phi_{\mathrm{cl}} /\left(\Phi_{\mathrm{cl}}+\Phi_{\text {bulk }}\right)$.

longer hold [see Fig. 2(b)]. Thus, the values of $\Xi$ in Fig. 3 likely underestimate the true dissipation ratio when $\mathrm{Ca}>0.02$. In the lower bound $(\mathrm{Ca} \rightarrow 0)$, the system would approach a depinning threshold, where the water-oil contact line would move by hopping between surface defects, resulting in a $\theta_{b}(\mathrm{Ca})$ relation that is very different from the generalized Cox equation [7-9]. The fact that the motion of the water-oil meniscus in our experiments appears to be smooth and $\theta_{b}$ is in good agreement with the generalized Cox equation suggests that we are either sufficiently far from the depinning threshold or that the strength of the surface defects on our glass surface is too small to have appreciable influence on the overall trend in Fig. 3.

The ratio of contact line to bulk dissipation in Fig. 3 has important macroscopic implications for problems beyond constant-rate imbibition. Neglecting dissipation near the contact lines would lead to erroneous (linear) relation between dissipation and $\mathrm{Ca}$; Fig. 3 demonstrates that this relation is nonlinear and is a function of the slug dimensions. One example where this may be significant is the flow of foam or ganglia in porous media [40,41], a system that inherently features a large number of (potentially very short) viscous slugs and thus might be expected to have significant energy dissipation associated with dynamic contact-angle effects. Another example is classical imbibition in capillary tubes. It has recently been demonstrated that early-time viscous effects near the contact line move the system away from the commonly known form of the Washburn equation $\left(z \sim t^{1 / 2}\right)$, towards $z \sim t$ [34]. This is when $\Phi_{\text {bulk }}$ and $\Phi_{\mathrm{cl}}$ are comparable. However, this flow regime is rather brief in classical imbibition (see Supplemental Material [38]). Alternatively, one can readily access the flow regime with significant $\Phi_{\mathrm{cl}}$ contribution through constant-rate imbibition, as we demonstrate in Fig. 3.

In summary, we have mapped out the contributions of contact-line and bulk dissipation during fluid-fluid displacement, and we have shown that a large portion of the dissipation takes place in the vicinity of the contact line. We did so using constant-rate spontaneous imbibition, achieved by introducing a viscous oil slug in front of the invading fluid inside a capillary tube. The rate of imbibition in such experiments can be precisely controlled through the viscosity and length of the oil slug. This setup allows probing flow regimes that would otherwise be accessible only during the early-time spontaneous flow - a novel feature of our experimental setup that has significant utility in the study of moving contact-line problems. Alternatively, one can ensure that dynamic contact-angle effects are negligible by making the oil slugs sufficiently long ( $\Xi \rightarrow 0$ when $l / R \gg 1$ ). For example, in order for contact-line dissipation to account for less than $5 \%$ of total dissipation, a slug must be longer than $l / R=$ 155 at $\mathrm{Ca}=0.02$ and longer than $l / R=65$ at $\mathrm{Ca}=0.2$.

The system we present in this work could be utilized for fabrication of precise micro- and nanopumps. The ability to precisely control the flow rate without external forces would be useful in designing passive microfluidic devices [42], which have applications in miniature heat pipes for cooling of electronic components [43], patterning biomolecules in microchannels [44], and clinical diagnostics [45]. Indeed, a known method of maintaining a fixed flow rate in such devices is by having a constriction ahead of the flow channel that is about an order of magnitude smaller than the rest of the channel [46]. However, it can be technically challenging to scale down this technique to sizes below a micron, where one would need to precisely fabricate nanometer-scale constrictions. The constant-rate imbibition depicted in Fig. 1(b) does not have such scaling limitations, and it is a cheap technique that can be used for passive control of flow rates in microfluidic devices.

We thank Lydia Bourouiba, John W. M. Bush, Philip M. Gschwend, and Jun Li for helpful discussions and suggestions. This work was funded by the KFUPM-MIT collaborative agreement "Multiscale Reservoir Science."

juanes@mit.edu

[1] M. Alava, M. Dubé, and M. Rost, Adv. Phys. 53, 83 (2004).

[2] J. Kim, M.-W. Moon, K.-R. Lee, L. Mahadevan, and H.-Y. Kim, Phys. Rev. Lett. 107, 264501 (2011).

[3] L. Fisher, Nature (London) 397, 469 (1999).

[4] P. G. de Gennes, Rev. Mod. Phys. 57, 827 (1985).

[5] J. H. Snoeijer and B. Andreotti, Annu. Rev. Fluid Mech. 45, 269 (2013).

[6] F. Brochard-Wyart and P. G. de Gennes, Adv. Colloid Interface Sci. 39, 1 (1992).

[7] J. F. Joanny and M. O. Robbins, J. Chem. Phys. 92, 3206 (1990).

[8] E. Raphaël and P. G. De Gennes, J. Chem. Phys. 90, 7577 (1989).

[9] P. Sheng and M. Zhou, Phys. Rev. A 45, 5694 (1992).

[10] J. De Coninck and T. Blake, Annu. Rev. Mater. Res. 38, 1 (2008). 
[11] A. A. Pahlavan, L. Cueto-Felgueroso, G. H. McKinley, and R. Juanes, Phys. Rev. Lett. 115, 034502 (2015).

[12] B. Levaché and D. Bartolo, Phys. Rev. Lett. 113, 044501 (2014).

[13] J. Eggers and H. A. Stone, J. Fluid Mech. 505, 309 (2004).

[14] J. C. Bird, S. Mandre, and H. A. Stone, Phys. Rev. Lett. 100, 234501 (2008).

[15] D. Bonn, J. Eggers, J. Indekeu, J. Meunier, and E. Rolley, Rev. Mod. Phys. 81, 739 (2009).

[16] C. Huh and L. E. Scriven, J. Colloid Interface Sci. 35, 85 (1971).

[17] S. Moulinet, C. Guthmann, and E. Rolley, Eur. Phys. J. B 37, 127 (2004).

[18] H. Perrin, R. Lhermerout, K. Davitt, E. Rolley, and B. Andreotti, Phys. Rev. Lett. 116, 184502 (2016).

[19] R. L. Hoffman, J. Colloid Interface Sci. 50, 228 (1975).

[20] M. Fermigier and P. Jenffer, J. Colloid Interface Sci. 146, 226 (1991).

[21] A. E. Seaver and J. C. Berg, J. Appl. Polym. Sci. 52, 431 (1994).

[22] L. H. Tanner, J. Phys. D 12, 1473 (1979).

[23] T. E. Mumley, C. Radke, and M. C. Williams, J. Colloid Interface Sci. 109, 398 (1986).

[24] E. Schäffer and P.Z. Wong, Phys. Rev. Lett. 80, 3069 (1998).

[25] E. Schäffer and P. Z. Wong, Phys. Rev. E 61, 5257 (2000).

[26] M. Hilpert, J. Colloid Interface Sci. 337, 131 (2009).

[27] M. Hilpert, J. Colloid Interface Sci. 344, 198 (2010).

[28] M. Heshmati and M. Piri, Langmuir 30, 14151 (2014).

[29] P. L. L. Walls, G. Dequidt, and J. C. Bird, Langmuir 32, 3186 (2016).

[30] O. V. Voinov, Fluid Dyn. 11, 714 (1977).

[31] R. G. Cox, J. Fluid Mech. 168, 169 (1986).
[32] A. Oron, S. H. Davis, and S. G. Bankoff, Rev. Mod. Phys. 69, 931 (1997).

[33] E. W. Washburn, Phys. Rev. 17, 273 (1921).

[34] J. Delannoy, S. Lafon, Y. Koga, E. Reyssat, and D. Quéré, Soft Matter 15, 2757 (2019).

[35] J. Bico and D. Quéré, J. Fluid Mech. 467, 101 (2002).

[36] J. Bico and D. Quéré, J. Colloid Interface Sci. 243, 262 (2001).

[37] B. Zhao, A. A. Pahlavan, L. Cueto-Felgueroso, and R. Juanes, Phys. Rev. Lett. 120, 084501 (2018).

[38] See Supplemental Material at http://link.aps.org/ supplemental/10.1103/PhysRevLett.125.174503 for details of the experimental setup, the generalized Cox equation, and energy dissipation in classical imbibition, which includes Ref. [39].

[39] W. Thielicke and E. J. Stamhuis, J. Open Res. Software 2, e30 (2014).

[40] K. T. Tallakstad, H. A. Knudsen, T. Ramstad, G. Løvoll, K. J. Måløy, R. Toussaint, and E. G. Flekkøy, Phys. Rev. Lett. 102, 074502 (2009).

[41] K. T. Tallakstad, G. Løvoll, H. A. Knudsen, T. Ramstad, E. G. Flekkøy, and K. J. Måløy, Phys. Rev. E 80, 036308 (2009).

[42] M. Zimmermann, H. Schmid, P. Hunziker, and E. Delamarche, Lab Chip 7, 119 (2007).

[43] L. L. Vasiliev, Appl. Therm. Eng. 28, 266 (2008).

[44] E. Delamarche, D. Juncker, and H. Schmid, Adv. Mater. 17, 2911 (2005).

[45] C. H. Ahn, J. W. Choi, G. Beaucage, J. H. Nevin, J. B. Lee, A. Puntambekar, and J. Y. Lee, Proc. IEEE 92, 154 (2004).

[46] W. Guo, J. Hansson, and W. van der Wijngaart, Microsyst. Nanoeng. 4, 2 (2018). 